\title{
Are causal analysis and system analysis compatible approaches?
}

\author{
Federica Russo \\ Philosophy, Louvain and Kent \\ f.russo@kent.ac.uk \\ To appear in International Studies in Philosophy of Science
}

\begin{abstract}
In social science, one objection to causal analysis is that the assumption of the closure of the system makes it too narrow in scope, that is it only considers 'closed' and 'hermetic' systems thus neglecting many other external influences. On the contrary, system analysis deals with complex structures where every element is interrelated with everything else in the system. The question arises as to whether the two approaches can be compatible and whether causal analysis can be integrated into the broader framework of system analysis. This paper attempts a negative answer on the grounds of fundamental differences in their assumptions and suggests using system analysis as a post hoc comparative tool.
\end{abstract}

Contents:

1. Descriptivist, causalist, and systemic approaches

2. Causal analysis

3. System analysis

4. Causal modelling vs. system analysis

5. Systemics: a post hoc comparative tool?

\section{Descriptivist, causalist, and systemic approaches}

The social sciences study societies and humans from different angles and perspectives. Think of economics, that studies the management of goods and services, or of demography, that studies variations in the population characteristics due to mortality, fertility and migration, or of epidemiology (half way between demography and medicine), that is interested in the distribution of disease within a population or across populations. Broadly speaking, two main approaches might be distinguished. Some social scientists are descriptivist, whilst others are overtly causalist. The former are interested in giving the best possible picture of a given social phenomenon, without, for a whole range of different reasons, daring any causal interpretation. The latter, conversely, take causal attribution and causal explanation to be an essential element for understanding, predicting and intervening on society. To be sure, there is a third approach. This is systemics. Systemics, or system analysis, takes it as a starting point that reality, at any level, is organised into systems, and that in a system every element is interrelated to everything else. In the social sciences, the goal will be to analyse social systems according to the principles of a general system theory.

Reconciling descriptivists and causalists seems to be a difficult task albeit not impossible. As a causalist, I should simultaneously work on two fronts: on the one side, to convince descriptivists that they do need causality (although, of course, mere descriptive knowledge is essential in a first exploratory stage), and on the 
other side, to improve causal methods and their foundations. Reconciling causalists and system theorists is also a hard and complex task. Here, I'll take up this second challenge.

The paper is organised as follows. I present causal analysis and system analysis in section 2 and 3, respectively. Attention is paid to their specific assumptions and to their methodology. I then try to show that the comparison of the two approaches poses a dilemma. If we take system analysis seriously, the two approaches clash too much to be compatible. If we do not, the two approaches are not so different, after all. Section 4 considers the first horn of the dilemma. I argue that causal and system analysis differ too much in their fundamental assumptions and methodology to be complementary, and that system analysis fails as an analytical tool. Finally, the last section considers the second horn and explores the possible role of system analysis as a post hoc comparative tool.

\section{Causal analysis}

\section{Different types of causal models and of goals}

Causal analysis is concerned with identifying causes and effects of social phenomena with the purpose of understanding, predicting and eventually intervening on society and on individuals. Thus, on the one hand causal analysis engages with a cognitive goal - this relates to explanation - and on the other hand it engages with a an action-oriented goal- this relates to inference and decision.

Typically, two types of causal analysis are distinguished: qualitative and quantitative. Qualitative analysis is performed by methods such as interviews or direct participation into settings. Unlike qualitative analysis, quantitative analysis takes advantage of the statistical machinery developed in the pioneering works of Wright in the 1920s, which grew in complexity and sophistication thanks to the work, to mention just a few, of Blalock and Duncan in the 1960s and 1970s, and more recently, since the 1980s, of Pearl and of Spirtes, Glymour and Scheines. Statistical models used for causal analysis are customarily called, 'causal models', at least since the 1960s. In spite of this apparent difference, the two types of causal analysis arguably share the same goals. However, to make the case for this claim would be the object of another paper, so here I shall confine the discussion to quantitative causal analysis in the social sciences. Quantitative causal models can also be of different types. Some aim to measure effects of causes (e.g., Rubin's potential outcome model), while others aim to disentangle causal mechanisms (e.g., structural equation and path models). Some aim to infer causal relations inductively from data, while others aim to confirm a hypothesised causal structure. In the following, I shall mainly consider causal models that aim to disentangle causal mechanisms using a hypothetico-deductive methodology, and shall focus on their goals, assumptions, and methodology.

\section{Quantitative causal analysis}

A causal model typically consists of a set of mathematical equations and/or of a graph laying down the hypothesised causal structure pictorially. More technical and 
precise definitions of causal model are of course possible. See for instance Wunsch (1988) and Freedman (2005) for an accessible introduction to causal modelling and statistics, Mouchart et al. (2008) and Russo (2008) for a critical evaluation of them. For the purpose of the present paper we can keep technicalities to a minimum. Typically, both equations and graphs are employed. However, one can start by writing the equations and subsequently drawing the graph or the other way round. Causal models normally consist of sets of equations, indicating a web of causal and non-causal relations among the variables of interest.

Causal models also rest on a number of assumptions, falling into three groups: statistical, extra-statistical, and causal. Among the statistical assumptions we find, for instance, linearity, normality, and non-measurement error. Those are standard statistical assumptions also made in associational models. However, causal models are provided with a much richer apparatus that allows their causal interpretation. In this apparatus we find background knowledge, the conceptual hypothesis, a number of extra-statistical assumptions and of causal assumptions. Among extrastatistical assumptions we can list the direction of time, causal asymmetry, causal priority, causal ordering. Causal assumptions include: no-confounding, noncorrelation of error terms, stability, and invariance. For a detailed account of the statistical, extra-statistical, and causal assumptions in causal models, see Russo (2008, ch.3-4). In the following, I shall focus on the assumption of the closure of the system and on the hypothetico-deductive methodology as they are most relevant for the comparison with system analysis.

\section{The closure of the system}

A major assumption of causal analysis is the so-called assumption of closure of the system. This assumption says that the system under analysis is not subject to external influences and thus can be separated, so to speak, from the larger web of interrelations in which it is located. Thanks to this assumption we can, at least in principle, detect the causes acting in the mechanism under investigation. As we shall see later in section 4 , in this assumption lies the bulk of the divergence between system analysis and causal analysis.

To be sure, two variants of the closure ought to be distinguished: strong and weak. Strong closure assumes that there is no influence at all in any of the variables figuring in the model, neither in the causes, nor in the effects. Pictorially:

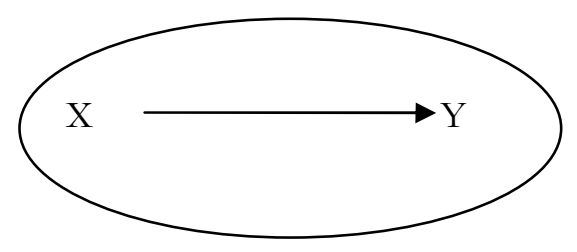

FIG. 1 Strong closure 
This assumption, however, is too strong and is normally relaxed in favour of weak closure: variables in the model undergo influences from non-observed variables non correlated between themselves.

Pictorially:

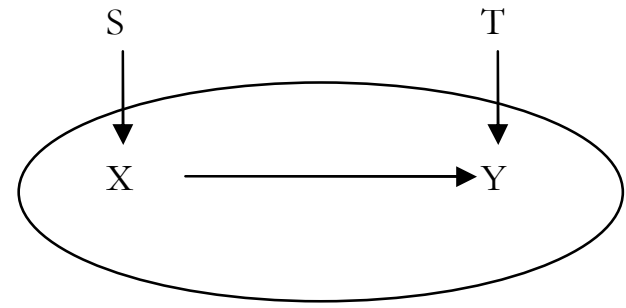

FIG. 2 Weak closure

Closure fails when non-observed variables influencing the variables in the model are instead correlated between themselves thus leading to a situation of confounding and making it impossible to estimate correctly the (causal) relation between the cause(s) and the effect.

The assumption of the closure is obviously related to covariate sufficiency and no-confounding. Simply put, covariate sufficiency says that the model includes all the variables needed in order to account for the phenomenon under investigation. Conversely, no-confounding says that variables liable to confound the relation between the 'true' cause(s) and the effect have been controlled for. Thus, if we put those three pieces of the puzzle together-(weak) closure, covariate sufficiency, and no-confounding - we end up with a causal model that describes a (quasi) hermetic mechanism where all and only the relevant variables play a role.

\section{Hypothetico-deductivism}

Causal models, at least those I'm concerned with in this paper, ${ }^{1}$ employ a hypothetico-deductive methodology. Simply put, hypothetico-deductivism is the view according to which scientists first formulate hypotheses and then test them by seeing whether or not the consequences derived from the hypotheses obtain. Popper (1959), who developed the H-D methodology, was motivated by the need of creating a scientific method in a non-inductive way. However, in causal analysis, hypothetico-deductivism takes a slightly different facet specifically concerning deduction, but does borrow from the Popperian account the primary role of the hypothesis-formulation stage. I shall get back to this point shortly.

\footnotetext{
1 As mentioned above, causal models can also be used in an inductive way, e.g. data mining. This is, for instance, the approach of Spirtes, Glymour and Scheines (1993), and, to some extent, of Pearl (2000). Inductivist approaches claim that causal relations can be inferred from data without the burden of extra-statistical and causal assumptions made in their hypothetico-deductive counterparts. However, it goes far beyond the goal of this paper to discuss the success of inductive causal models. Consequently, the scope will be limited to causal models that employ a hypotheticodeductive methodology.
} 
According to the H-D methodology, model building and model testing essentially involve three stages:

1. formulate the causal hypothesis;

2. build the statistical model;

3. draw consequences to conclude to the empirical validity or invalidity of the causal hypothesis.

The hypothesis to put forward for empirical testing does not come from a tabula rasa, but emerges from a causal context, namely from background knowledge, that is knowledge concerning the phenomenon at stake, previously established theories, and preliminary analyses of data. The causal hypothesis, that states a hypothesised causal mechanism, is also called the 'conceptual hypothesis' and is not analysable a priori. That is to say, its validity is not testable by a logicolinguistic analysis of concepts involved therein. On the contrary, to test the validity of the causal hypothesis requires building a statistical model, and then drawing consequences from the hypothesis in order to test the hypothesis against empirical data.

The estimation of the statistical model and hypothesis testing will allow us to conclude to the empirical validity or invalidity of the causal hypothesis. If the model is correctly estimated and fits the data, and if certain conditions are satisfied (notably, exogeneity and invariance) the hypothesised causal link is accepted, rejected otherwise. The hypothetico-deductive structure of causal modelling is thus apparent: a causal mechanism is first hypothesised and then put forward for empirical testing. That it to say, the causal hypothesis is not directly inferred from the data gathered, as is the case with inductive strategies, but accepted or rejected depending on the results of tests and on background knowledge.

As anticipated above, hypothetico-deductivism in causal modelling does not involve deduction structu sensu, but involves a weaker inferential step of 'drawing consequences' from the hypothesis. Once the causal hypothesis is formulated out of the observation of meaningful co-variations between the putative cause(s) and the putative effect and out of background knowledge, we do not require data to be implied by the hypothesis but just that data conform to it. Here, 'conform' means that the selected indicators and relations among them adequately represent the conceptual variables appearing in the causal hypothesis and the relations among them. Thus, this way of validating the causal hypothesis is not, strictly speaking, a matter of deduction, but surely is, broadly speaking, a deductive procedure. More precisely, it is a bypothetico-deductive procedure insofar as it goes the opposite direction of inductive methodologies: not from rough data to theory, but from theories to data. For a discussion of the H-D methodology, see also Little (1998, ch.9), Cartwright (2007, ch. 2) and Russo (2008, ch. 3).

\section{Modelling mechanisms}

I just said that hypothetico-deductive causal models aim to (dis)confirm a causal hypothesis, and only hurriedly mentioned that the causal hypothesis is about a causal mechanism. Let me develop this idea a bit further.

What do causal models do? Causal models model the properties of a social system. In particular, they model the relations between the properties or 
characteristics of the system, which are represented by variables. By 'social system' I simply mean, for the time being, a given population. 'Population', in turn, has to be understood here in the statistical sense, that is as a set of units, those units being individuals, households, firms, etc.

To model the properties of a social system means to give the scheme, or the skeleton, of how these properties relate to each other. However, this causal mechanism is not modelled in terms of spatio-temporal processes and interactions à la Salmon (1984 and 1990) but is statistically modelled. Concepts typical of statistical causality-e.g., statistical relevance, comparison of conditional probabilities, and screening-off-are used in order to identify the types of relationships that hold among the variables of interest. This is how the causal model models the causal mechanism governing the social system. In particular, causal models seek to uncover stable variational relations between the characteristics of the system. For one account of the notion of variation and of mechanism in causal modelling, see Russo $(2008$, ch.6). For the present discussion, we need to keep in mind the following points: (i) mechanisms can be conceived of as schemes of how variables relate to each other; (ii) in a mechanism, variables play specific (causal) roles, e.g. direct, indirect, or intermediate causes; (iii) some types of relations in causal mechanisms are excluded, e.g. instantaneous feedback loops ${ }^{2}$.

\section{System analysis}

\section{Scope and goals of system analysis}

Under the label 'system analysis', or 'systemics', or 'systemism', falls the general approach to systems presented in the works of e.g. von Bertalanffy (1968), Simon (1969), and Bunge (1979).

The systemic viewpoint becomes necessary, system theorists say, for a number of reasons. For instance, various scientific disciplines encountered difficulties espousing causalist and/or positivistic approaches. But mostly, as systems are ubiquitous and arguably systems are exactly the kind of thing they study, a general framework is needed.

More specifically, a general system theory is, according to von Bertalanffy, a theory the subject matter of which is the formulation and derivation of those principles which are valid for systems in general. In the Preface, von Bertalanffy defines system science the scientific exploration and theory of system in the various sciences. He indicates the major aims of general system theory thus (von Bertalanffy 1968, p. 37):

This indicates major aims of a general system theory:

1. there is a general tendency towards the interpretation in the various sciences, natural and social;

2. such interpretation seems to be centred in a general system theory;

3. such theory may be an important means of aiming at exact theory in the non-physical fields of science;

\footnotetext{
${ }^{2}$ Feedback loops that are ordered in time are admissible: $X_{t}$ causing $Y_{t}$, which in turn causes $X_{t}$, but at a later time.
} 
4. developing unifying principles running 'vertically' through the universe of the individual sciences;

5. this can lead to a much-needed integration in scientific education.

In the same vein, Bunge (1979) conceives of system analysis as a set of theories that focus on the structural characteristics of systems. Therefore systemism aims to cross the largely artificial barriers between different disciplines. Thus Bunge qualifies system theory as having two characteristic traits: (i) there are some concepts and structural principles that seem to hold for systems of many kinds, and (ii) there are modelling strategies that seem to work everywhere. Furthermore, according to Bunge, systemics has two main motivations: cognitive and practical. The cognitive motivation is the wish to discover similarities among systems of all kind despite the different contexts in which they arise and operate. The practical motivation amounts to the need to cope with the huge and many-sided system characteristic of industrial society.

\section{Systems and the whole}

What is a system, then? Informally, a system is a set of elements standing in reciprocal interrelation. Somewhat more formally, as von Bertalanffy (1968, p. 37) states:

Elements, $p$, stand in relation, $R$, so that the behaviour of an element $p$ in $R$ is different from its behaviour in another relation, $R$ '. If the behaviours in $R$ and $R$ ' are not different, there is no interaction, and the elements behave independently with respect to the relations $R$ and $R$ '.

This is still a qualitative characterisation of what a system is. Systems are mathematically defined by certain families of differential equations, and properties of those systems are found as long as more specific conditions are introduced. I won't linger on the mathematical technicalities of the definition of system, for which I direct the reader to von Bertalanffy (1968, ch. 3) and Bunge (1979, ch. 1).

It will be worth distinguishing, from the very beginning, between aggregates and systems. Unlike systems, aggregates are just collections of items not held together by bonds and therefore lacking any integrity or unity (see Bunge 1979, p. 4). However, both systems and aggregates can be either concrete or conceptual. An example of conceptual system is a scientific theory and an example of concrete system is a school.

It is worth noting that Bunge (1979, p. 2) also draws a distinction between systemics and system analysis. System analysis studies many-sides and multi-level systems and for doing so it must adopt various points of view on different levels. However, unlike systemics, system analysis is not particularly interested in deemphasising the peculiarities of the components of the system to the benefit of the interrelations that hold in it. Nevertheless, other authors, including von Bertalanffy, use the two terms as synonyms meaning the precise sense of 'systemics'. A corollary of this distinction is that Bunge would possibly locate multilevel or hierarchical models, a particular class of quantitative causal models, within system analysis but not necessarily in systemics. In other words, the 
recognition of a multi-sided and multi-level organisation of reality is not sufficient for systemics.

Systemics is the science of the whole. Traditionally, the science of the whole comes in two variants, namely holism and atomism. In a nutshell, holism is the ontological view that stresses the integrity of systems at the expenses of their components and of the mutual actions among them. On the other hand, atomism is the view that the whole is contained in its parts, so that the study of the latter should suffice for understanding the former. However, Bunge contends that these two doctrines be capable of properly analysing systems (Bunge 1979, p. 39ff). Instead, what makes systemics a successful tool for the science of the whole is its proper methodology:

1. the identification of the components of the system,

2. the identification of the environment, and

3. the identification of the structure.

Indeed, in a systemic perspective, it makes little or no sense at all to hypothesise any relations without knowing what the relata may be. This, it goes without saying, is in sharp contraposition with the H-D methodology of causal modelling. But I shall get back to this point in section 4 .

\section{Systemics: a different worldview}

The starting point of the systemic approach is that the world shows a systemic organisation, that is systems, as said above, are ubiquitous-living beings organise themselves in systems at the biological as well societal level. Thus, the concept of system becomes omnipresent in virtually all scientific domains. This, as a simple recognition about the world would be rather innocuous-yet true-if it were not accompanied by a much stronger assumption that in a system every thing is interrelated with everything else.

In fact von Bertalanffy (1968, p. 16), in Kuhnian terms, thinks that system analysis opens altogether a new paradigm. Various systemic approaches may be logically inhomogeneous and represent different conceptual models, mathematical techniques or point of views; nonetheless they have a common denominator in being system theories. This common denominator is constituted by the specific concept of system thereby developed.

Let me now draw the reader's attention to the most controversial foundational aspects of systemics. A philosopher would call them the philosophical foundations, a scientists may content herself with worldview. Either way, these are the assumptions at the very basis of the general system theory and without them it does not make any sense to call an analysis systemic.

von Bertalanffy (1968, p. xvi) explicitly talks about a system philosophy. This is a reorientation of thought and worldview ensuing from the introduction of 'system' as a new scientific paradigm. In particular, this turn goes against the traditional analytic, mechanistic and one-way causal paradigms of classical science. Unfortunately, very little space is devoted in his book to the discussion of the system ontology and epistemology and to the comparison with the (positivist) philosophy that underlies classical science. A main issue with system ontology is 
the nature of systems. Some systems are real (e.g., a galaxy, an atom), others are conceptual (e.g., maths and logic systems), and others are abstract (e.g., scientific models). But those distinctions are not as clear-cut. A social or ecological system, for instance, is real and yet it is not the object of direct observation and perception by an observer or agent. The commonsense distinction between what is 'real' and what is 'conceptual' thus lacks a meaningful counterpart in systemics-the issue, as von Bertalanffy acknowledges, deserves further investigation and clarification. System epistemology also breaks down a pillar of the 'received' (and commonsense) epistemology, namely the distinction between the object of knowledge and the knowing subject. As von Bertalanffy says (1968, p. xx):

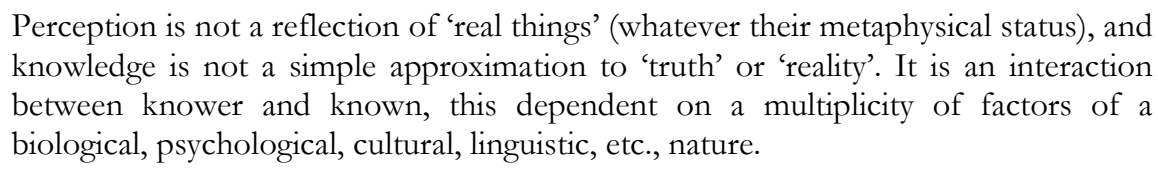

However, the most relevant philosophical shift is the following (von Bertalanffy 1968, p. $\mathrm{xxi})$ :

\begin{abstract}
The third part of systems philosophy will be concerned with the relations of man and world or what is termed 'values' in philosophical parlance. If reality is a hierarchy of organized wholes, the image of man will be different from what it is in a world of physical particles governed by chance events as ultimate and only 'true' reality. Rather, the world of symbols, values, social entities ad cultures is something very 'real'; and its embeddedness in a cosmic order of hierarchies is apt to bridge the opposition of C. P. Snow's 'Two Cultures' of science and the humanities, technologies and history, natural and social sciences, or in whatever way the antithesis is formulated.
\end{abstract}

von Bertalanffy is denying the distinction between knower and known. The knowing agent belongs to the system, she interacts with it, and therefore changes it in the process of knowing it. von Bertalanffy aims to bridge Snow's 'Two cultures', thus contributing to the increasing constructivist worldview in the humanities and extending it to science by claiming scientific method to be embedded into language and culture and therefore lacking objectivity. On the contrary, classical science claims that the observer can still have a grip on reality by making objective and non-culturally embedded statements about the world.

Bunge also devotes a chapter to the 'systemic worldview', as he calls it. To begin with, Bunge $(1979$, p. 245) warns us not to confuse systemics with the 'popular' system philosophy which is a new version of holism according to which everything is a system and the patterns of being and becoming are basically the same at all levels. Both these claims, in the general system theory, do not hold.

Bunge articulates a complex systemic worldview around assumptions, postulates, and theorems. How radical is this worldview will become now apparent. The first assumption is that there are no stray things. Every thing interacts with other things so that all things cohere in forming systems. From which, the postulate follows (Bunge 1979, p. 245): every concrete thing is either a system or a component of one. Systems, adds Bunge, come into Chinese boxes or nested systems. Bunge then states that the following generalisations follow from 
the assumptions and from the axioms about things and change he developed elsewhere (Bunge 1977):

1. every system is engaged in some process or other;

2. every change in a system is lawful;

3. because every subsystem acts upon or is acted on by other subsystems, it exists per alium rather than autonomously;

4. the universe as a whole exists per se and, moreover, it is the only absolute (autonomous) existent;

5. the universe has neither beginning nor end in time: it endures although no part of it does.

For a concise exposition of Bunge's thought on systemics, see also his (2000). It is not my intention to engage with a deep metaphysical discussion about the autonomous existence of the universe. The first two generalisations are controversial enough and offer us enough matter for a comparison between the systemic and causalist approaches.

The consequences of such a different perspective on reality are expressed very clearly by Mingers (2006), who seeks to apply systemics to the field of business and management. He says (Mingers 2006, p. 3):

\footnotetext{
If you follow this path [i.e., system methodology] to its logical conclusion then you end up in a solipsistic pit from which it is difficult to escape. Every theory becomes simply another viewpoint or Weltanschaung, another interpretation of the world, no better no worse than any other. There can be no external social world that enables or constraints us, indeed no world at all that is more than a construction of the observer.
}

This, of course, would make any scientific enterprise impossible, if Mingers did not point to the way out, that is critical realism. This view, that he attributes to Roy Bhaskar, basically acknowledges the reality of the world and of its objects (i.e., they are mind-independent) but at the same time holds that knowledge, especially in the social sciences, is always fallible and cultural, spatially and historically relative.

Later, Mingers (2006, p. 257) claims that system thinking brings to the fore issues about epistemology, ontology, methodology, and ethics; those issues, he claims, recognise the primary role of the observer in any analysis. Minger accuses much academic research to carry on with little attention to those issues and then echoes Maturana who believed that "everything said is said by an observer" and Churchman who thought that "the system approach begins when you first view the world through the eyes of another". But he also goes on in saying that Positivism saw the effacement of the observer as one of the main planks of objectivity until Heisenberg demonstrated the inseparability of the observer and the observed even in the depth of quantum physics.

It seems to me that two morals could be drawn out of this. The first is that if we take systemics seriously, it altogether entails a radically different perspective on reality and on the way we have to make sense of it. This different worldview is in sharp contraposition with the metaphysics and epistemology underlying causal modelling. The second is that if the whole point is to recognise that knowledge is relative, then the question arises as to whether it is absolutely necessary to buy systemics with all its metaphysical burden. Moreover, to avoid solipsistic attitudes, Minger suggests endorsing a form of critical realism. However, doesn't this tenet 
eventually coincide with any other moderate realist stance in science? After all, no causal modeller (and perhaps nor any scientist) would claim that she will discover eternal and immutable truths.

\section{Social systems}

Both von Bertalanffy and Bunge present and discuss examples of systems in various disciplines, ranging from chemistry to physics, from anthropology to sociology. There are various methodological approaches to the study of systems. von Bertalanffy mentions, in the preface of his 1968, information theory, cybernetics, game theory, decision theory, stochastic models, etc.

von Bertalanffy acknowledges that these are rather inhomogeneous approaches, representing different conceptual models, mathematical techniques and even different general points of view; however, they are in accord in being 'system theories'. The extent to which some of the aforementioned approaches are rightly put into the systemic approach, I will not thoroughly investigate. Let me just note that standard approaches in e.g. decision theory or game theory do not presuppose the heavy metaphysics of systemics, namely that every thing is either a system or a component of it and that in a system every thing interacts with everything else. So it is not clear whether decision and game theorists have been system theorists without being aware of, or, after all, systemics does not carry an altogether different worldview. But I shall discuss this point more thoroughly in the last section.

von Bertalanffy (1968, ch. 8) considers the case of the social sciences. He conceives of them quite broadly as to include sociology, economics, political science, but also cultural anthropology or linguistics. The social sciences are, in his view, the sciences of systems par excellence. A systemic view in this field goes against atomistic conceptions, which neglect the study of relations, and against conceptions that neglect the specificity of the systems concerned, such as 'social physics'. It is worth noting that von Bertalanffy does not explicitly mention Adolph Quetelet's social physics. However, the reference to Quetelet seems a quite straightforward interpretation as the critical target is a conception that would reduce complex social interrelations to deterministic physical ones.

Bunge (1979, ch. 5) develops systemics in the social sciences in more detail. A systemic analysis in the social sciences starts with some assumptions about what society is. A human society is, at bottom, an animal society but with novel properties; in particular, Bunge says, Man is faber and sapiens, oeconomicus and politicus, artifex and ludens. These characteristics will also determine the type of relations and activities that take place in human societies. As any other systems, human society is defined depending on the following three elements: composition, environment, and structure. A buman society is composed by humans who share an environment and transform it deliberately; they hold social relations and communicate among themselves, they are divided into social groups and constitute a self-reliant limit. It is worth noting that, according to the systemic view, a factory, a school or an army are indeed subsystems of a society but are not societies themselves. Societal properties are also formally defined (see Bunge 1979, pp. 190191). It will suffice to mention here that two types of properties are distinguished: 
resultant and emergent. A societal property $P$ of a society $\sigma$ is a resultant property if, and only if, $P$ is also a property of some components of $\sigma$, otherwise it is an emergent property. For instance, the total food consumption per year is a resultant property as it is a mere aggregation of the individual consumptions. Social differentiation, participation or cohesion are, instead, emergent societal properties.

Another characteristic trait of society is that they are composed by various interdependent subsystems, in particular the kinship, economic, cultural and political. The systemic analysis of social systems aims at describing the structure of society and keeping track of its changes. Bunge gives an accurate account of the internal systemic dynamics of social subsystems and systems.

To sum up, system analysis aims at describing the complex structure of systems in various scientific domains under two major assumptions: (i) there is no sharp distinction between knower and known, and (ii) every thing is interrelated with everything else. However, a part from the generic methodological precepts of identifying in this order (i) the components, (ii) the environment, and (iii) the structure of the system, and of the mathematical description of the system in terms of differential equations, we do not find, in system theorists, a more detailed explanation of how to perform the analysis of systems, when it comes to analyse data sets.

\section{Causal modelling vs. system analysis}

\section{Complementary approaches?}

As mentioned at the end of sec. 1, the comparison of the two approaches seems to lead to a dilemma. If we take systemics seriously-in particular, we buy its metaphysics - the clash with causal analysis is too big to make them compatible with each other. If, instead, we grant system analysis a charitable interpretation, then the difference with causal analysis seems to vanish. I shall discuss the two horns of the dilemma in this and in the next sections, respectively.

Consider systemics with its metaphysics first. Causal modelling and systemics diverge on some crucial fundamental points. First, causal analysis assumes and relies on the distinction between knower and known, while in system analysis the observer is part of the system. This marks an important difference between the two approaches. As we have seen before, according to von Bertalanffy, the denial of the knower-known distinction is a fundamental element of the change of worldview. Causal modellers would agree that, especially in social contexts, objectivity is more a regulative concept than a target that can be practically achieved. No doubt social scientists are driven by their own backgrounds, traditions and prejudices when they analyse social phenomena, and this may affect the analysis. However, this is to say that the agent interprets the system, not that she changes it altogether while she studies it. The claim that the researcher changes the system she studies seems to be wrong in almost all social science research contexts. How can the social scientist possibly change the system if she is provided with observational data that she cannot manipulate in any possible way? Here the danger seems to concern whether the interpretation the social scientists provide 
will meet a (reasonably) good standard of objectivity. Agreed, when the social scientist (say, an anthropologist) is involved in field work or when she prepares and runs herself interviews, she does interact with the system, but this does not imply that that the distinction knower-known ceases to hold.

Second, causal analysis, unlike systemics, relies on the assumption of the closure of the system. Causal analysis isolates a mechanism within a larger context and attempts to detect the causal relations that take place therein. The challenge is exactly to make a selection among all the elements and relations existing in a given context. The selected elements and causal relations will then be exploitable for the purposes of explanation, prediction and intervention. The causal modeller cannot assume, as the system analyst does, that in a system every element is interrelated with any other element. This claim would be useless for explanation (it is a trivial—yet true_claim of any complex phenomenon) and for policy (it does not provide a criterion to choose which relations to intervene upon).

Third, in causal analysis mechanisms are established using prior information. Background knowledge plays here a fundamental role because it is based on this prior and often non mathematised or formalised information that researchers formulate the causal structure to test. On the contrary, system analysis is interested in the elements of the system and in their reciprocal interrelations abstracting from any prior information we might have. The whole context is given once we identify the elements and their relations. This is in sharp contraposition with the causal modelling practice. Any choice of variables that is independent of the context is considered arbitrary and unjustified. Agreed, the prior identification of the context would be no guarantee of the objectivity of the research procedures and of the results, in the sense that disagreement among different social scientists may nevertheless arise. However, the specification of the context justifies the selection of variables and relations, not the other way round, as is the case in systemics.

There are also issues concerning determinism-Bunge says systemics holds determinism but not causalism-but I shall leave them aside. The three points just discussed, far from being minor differences, seem to make causal analysis and system analysis opposite approaches. And yet, the need to combine or integrate causal analysis and system analysis comes from different quarters. Here is one example.

Complementarity between the two types of analyses is promoted, for instance, by Franck (1994, 2002, 2007). Franck's motivation is a serious methodological difficulty of causal modelling. Often, it is very hard to identify and separate out causes and effects. Some cases are of course more difficult than others. For biological mechanisms, which are embedded in time, the task is pretty easy: smoking at time $t$ causes lung cancer at $t^{\prime}$, but not the other way round. However, when the temporal order of the variable is not available and/or when causal relations make sense in both directions, the task is much harder. For instance, we might hypothesise that migration causes marriage dissolution as the former is observed before the latter. This causal relation would make sense; however, it might be eventually disproved because, say, we overlooked a temporal prior process-e.g. marital problems and the subsequent decision to divorce-causing migration. To be sure, this is a problem concerning the available data rather than a 
conceptual problem. But system analysts claim that systemics does not incur into such problems because it relies on the separation parts/whole and seeks to identify the action of the parts on the whole and on the whole on the parts. This means that in a systemic analysis of the fictitious migration-marriage example above relations in both directions would be allowed with no apparent contradiction.

Franck (2002) proposes a general methodology that incorporates both system analysis and causal analysis. This methodology proceeds in the following steps (Franck 2002, p. 295):

(1) Beginning with the systematic observation of certain properties of a given social system, (2) we infer the formal (conceptual) structure which is implied by those properties. (3) This formal structure, in turn, guides our study of the social mechanism which generates the observed properties. (4) The mechanism, once identified, either confirms the advanced formal structure, or indicates that we need to revise it.

Step (1), in Franck's proposal, corresponds to the identification of the elements and of the environment in Bunge's methodology. Step (2) is the identification of the system within which we model causal mechanisms in steps (3) and (4). Then, steps (3) and (4) correspond to causal analysis as presented in sec. 2. This way of combining causal analysis and system analysis also finds application, according to Franck, in econometrics (see Franck 2007).

\section{Systemic and causal stories}

But let us compare causal analysis and system analysis using a real case study discussed by Michel Loriaux, a demographer that also advocates complementarity of the two approaches. Loriaux (1994) denounces a number of theoretical weaknesses of causal modelling: the choice of variables and indicators, the translation of variables into concepts, and the formulation of causal hypotheses. Among the features of causal modelling, Loriaux finds the assumption of the closure of the system particularly worrying. Recall, this assumption says that the mechanism that has been isolated constitutes a hermetic system which is not subject to external influences. This is what allows us to claim that (at least in principle) a variable is a cause of another if certain conditions hold, e.g. correlation, temporal priority, invariance, etc. This assumption of strict closure is not a realistic one, though. The way out is to admit that systems be partially permeable and that explicit variables can undergo influences of implicit variables. This is the weak closure of the system mentioned in sec. 2 .

However, this isn't enough. Loriaux takes as an example the causal model developed by Lopez-Rios et al. (1992). In this causal model researchers are interested in the effects of socio-economic development and of use of sanitary infrastructures on regional mortality in Spain. Spain met deep socio-economic changes in the mid-Seventies, and consequently policy in that period simultaneously tried to intervene to improve the social and economic situation. This led to a low mortality rate at the time of the study. It is this background that justifies the choice of distinguishing the supply and demand of medical care, unlike the majority of similar ecological studies. In fact, previous studies in demography and medical geography examined the incidence of the health system on regional 
mortality coming to the conclusion that regional differences in mortality could not possibly be explained by regional differences in the health system. Instead, here researchers (successfully) test the causal hypothesis that regional mortality is influenced by the health system which is in turn influenced by the social and economic development.

The causal model consists of the following graph and equations.

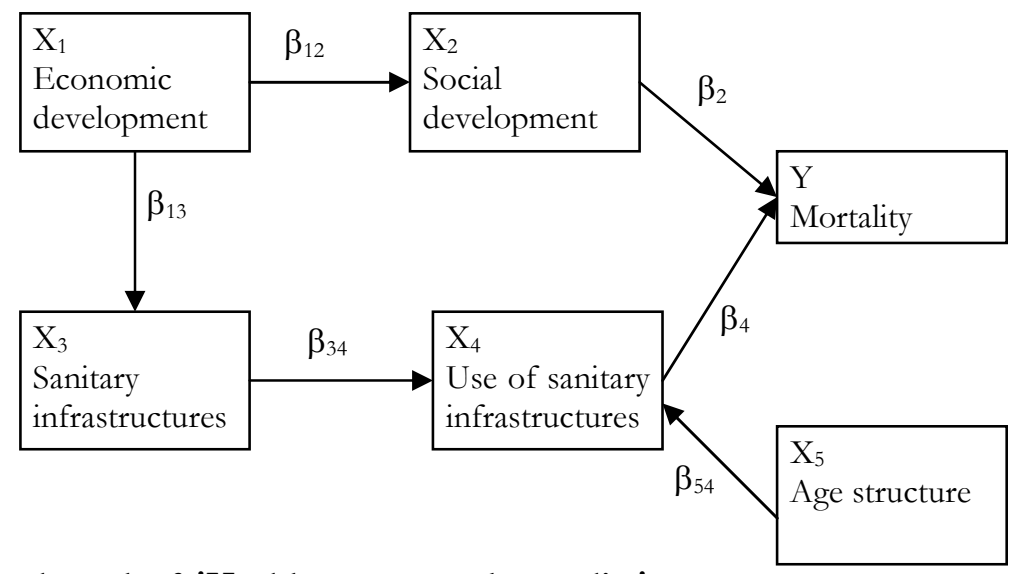

FIG. 3 Causal graph of 'Health systems and mortality'

$Y=\beta_{2} X_{2}+\beta_{4} X_{4}+\varepsilon_{1}$

$X_{2}=\beta_{12} X_{1}+\varepsilon_{2}$

$X_{3}=\beta_{13} X_{1}+\varepsilon_{3}$

$X_{4}=\beta_{34} X_{3}+\beta_{54} X_{5}+\varepsilon_{4}$

In this model, each variable is regressed on its immediate ancestors. $X_{1}$ and $X_{5}$ are exogenous, thus meaning that they are the causes of mortality and the other variables are intermediate variables, having a causal role just as effects of $X_{1}$ and $X_{5}$ but not on their own. In plain English, the equations state that regional mortality is causally determined by social and economic development; sanitary infrastructures alter mortality rate through the use of infrastructures, but the use of infrastructures depends on economic development, so it is not an exogenous cause; use of sanitary infrastructures, in turn, depends on the age of individuals and on 'sanitary infrastructure', so again it is not an exogenous cause, but it has causal impact on mortality only through other factors.

The first concern Loriaux has is that the principal variables Lopez-Rios et al. use, i.e. economic development, social development and sanitary infrastructure, are theoretical abstractions. Surely they correspond to real situations, yet they are difficult to grasp. The second concern is that the underlying assumption of this causal study is that, according to some economic and sociological theories, economic development generates social development. Few people would reject this claim, yet such a causal relation is full of other non-explicit assumptions and of simplifications that are sometimes hard to justify. Moreover, counterexamples exist, as there are situations in which variations in economic development are not followed by variations in social development, and even some people would reverse the causal arrow. This leads us to two problems. The first is the specification of 
the direction of the causal relation, but, most importantly, we face the problem of specifying the inference process.

As Loriaux correctly points out, this constitutes a serious setback for the action oriented goal of social research. If causes and effects are confused, i.e. they are not correctly specified, interventions won't have the expected impact. That is to say, to intervene on an cause which is not in fact a cause, won't deliver the results planned in the policy because causal relations are non-reversible. This is one of the basic principles of causal analysis. And if causal analysis cannot fully justify each step of the modelling procedure, then it is in serious trouble. The problems raised by the closure of the system show, according to Loriaux, that this is more than an assumption (to test), this is a postulate. This makes causal analysis too narrow in scope, thus overlooking the many interrelations between the elements of the system and failing to give the correct complex dynamics of the social system under analysis. Causal analysis, he claims, is undermined at the very basis, causal relations are chimeras and causal principles lead us to vicious circles.

Even if we want to dismiss causal analysis on these grounds, the question, Loriaux admits, still remains: how can we make sense of statistical covariations between variables if we abandon the causal framework? The solution resides in system analysis. Loriaux advocates a complementarity between the two frameworks rather than a complete replacement. One of the main properties of systems, were they social or biological, is of being homeostatic. This is the capability of a system to keep itself in a stable state by means of regulatory interdependent mechanisms, in spite of disturbing external influences and of the continuous regeneration of its components. When internal and external influences become too strong, those regulatory and control 'devices' can't keep the previous equilibrium anymore and this leads to changes in the system towards new functioning modes and different forms of structural organisation. During the processes of balancing, the components of the system can jointly evolve and those joint evolutions are exactly the covariations we consider to be causal. That is to say, Loriaux suggests that causality finds its domain of application within the broader systemic view.

Fig. 4 is the systemic story Loriaux provides for the same phenomenon LopezRios et al. (1992) analysed. This graph perhaps gives a more accurate and faithful picture of the phenomenon. I am concerned with Loriaux's methodology, though. Is it a quantitative or a qualitative method? It is systemics. Fair enough. Then, what are the differential equations that describe this system? How do we decide what variables have to be included? What is the theoretical justification of the relations? Many boxes contain groups of concepts-how are those conceptual variables measured? It is not hard to see that this systemic graph violates the basics rules of graphs used in causal modelling. In particular, many relations are double edged and there is no causal ordering. But this is a systemic story not a causal one, so fair enough. However, how do we set policies at all if all the elements in the system are interrelated? These are sensible questions any causalist with an interest in methodological issues would ask. I think there is a more serious problem in Loriaux's story, though. Loriaux criticises Lopez-Rios et al.'s causal story on the grounds that background knowledge suggests that the picture they provide is oversimplified and therefore incorrect. The same background knowledge, instead, 
suggests a more complex picture-the systemic story given in fig.4. Apparently Loriaux is using prior information to figure out what the systemic structure is. Thus the context, contrary to Bunge's methodological precepts, does play a major role to determine the structure, be it systemic or causal. It seems to me that not only causal analysis and systemic analysis clash too much in their fundamental assumptions, but that systemic practice contradicts its theory.

In sum, my systemic worries can be summarised thus. First, systems become very easily intractable and of difficult use for policy, which is nevertheless a major goal of causal modelling. Second, no precise and detailed methodology is offered, as of today, to analyse data sets. Last (but not least), assumptions clash to much to make those approaches compatible.

\section{Systemics: a post hoc comparative tool?}

The discussion above cast doubts on systemics as an analytical tool and somewhat excluded that causal analysis could be successfully incorporated into systemics. In the following, I will explore the second horn of the dilemma: what happens if we buy systemics without the burden of its metaphysics? What role, if any, is then left to systemics?

Let us start from what we have established so far. Systemics is not simply a different methodology, it is altogether a different approach to knowledge and reality. It is accompanied by a different metaphysics, without which we cannot say, strictly speaking, that we make systemic science. The question then arises as to whether it is plausible and/or necessary to buy its metaphysics. There are two reasons why it is not. The first is that if the goal is to recognise the relative character of knowledge and its unavoidable subjective component, then we definitively don't need to: contemporary science is liberated by some Positivistic totes. The second is that buying this metaphysics leads to an analysis of a given phenomenon that is of difficult use for policy reasons.

Having given a negative answer to this first question, a second one arises: is it possible to make systemic science without its metaphysics? Before giving my two cents, I shall consider the answer coming from systemics itself.

Since the pioneering works of von Bertalanffy, systemics has evolved. System science subsequently moved from a hard system thinking, to a soft system thinking, to a critical system thinking (see for instance Flood and Jackson (1991)). Whilst the idea that system thinking provides an altogether different worldview is still a recurring theme, the burden of the systemic metaphysics has been slowly abandoned. In critical system thinking, for instance, the observer is not part of the system anymore. Witness Checkland (1995, p.100):

Such thinking starts with an observer/describer of the world outside ourselves who for some reason of his own wishes to describe it 'holistically', that is to say in terms of whole entities linked in hierarchies with other wholes. This leads to the most basic prescription of what the observer's description will contain: the purpose, the system(s) selected, and various system properties such as boundaries, inputs and outputs, components, structure, the means by which the system retains integrity, and the coherency principle which makes it defensible to describe a system as a system. 
In various books on system science (see for instance Deutsch (1983), Ruberti (1984), Mingers (2006), Zgurovsky and Pankratova (2007)) there is no reference to the metaphysics that accompanied system thinking of the forefathers, and yet systemics is said to have its own specificity and novelty with respect to 'classical' science.

I rejoin Ruberti (1984), who in the introduction points out that system thinking gave an impetus to new fields of research, especially in differential equations and algebra. However, the question is whether this opens a new scientific paradigm, and to what extent this paradigm is significantly different from the old one. It seems to me that once the metaphysics of hard system thinkers is abandoned, system analysis loses its peculiarities, in particular those that supposedly make it a new scientific paradigm. Deutsch (1984, p. ix), for instance, claims that

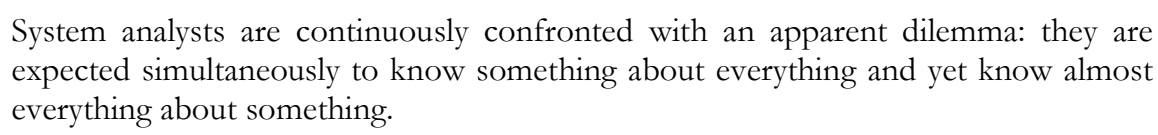

Why is this situation peculiar to system analysts? Don't causal modellers face the same problem? Deutsch does not provide a precise definition of 'system' (unlike hard system thinkers) but attempts a working definition of 'system analysis': on the one hand, it is a separation of a whole into its components parts, and, on the other hand, it is an examination of a complex structure, of its elements, and of their relations. Many of the techniques Deutsch presents to analyse systems are not specifically 'systemic': loss and risk functions, standard estimation techniques, Neyman-Pearson hypothesis testing do not have anything intrinsically 'systemic'. So, where is the specificity and novelty of system science?

Following up on system methods and applications, Aoki (1984) presents dynamic models in economics from a systemic perspective, but eventually his 'systemic' models do not differ from the 'classical' models. The only difference is a systemic 'manifesto' given in the opening of the paper (Aoki 1984, p. 113):

Broadly speaking, systems science can contribute in two related ways: by providing an alternative conceptual framework for dynamic analysis in areas where static or comparative static analysis is a dominant mode of analysis and by developing new tools to facilitate dynamic or comparative dynamic models.

Unfortunately, the alternative conceptual framework is not really provided, nor the new tools are incompatible with the tools of 'classical' science. So where is the change of paradigm?

The new paradigm ought to be accordingly accompanied by a novel methodology and possibly with novel theoretical principles guiding applied research. This is indeed the goal of Zgurovsky and Pankratova (2007). The 'metamethodology' of systems is concerned with those principles that are valid for systems in general, as von Bertalanffy said as early as 1968. In settling down the basic principles of system thinking, Zgurovsky and Pankratova (2007, p. 5) say:

System thinking is a higher form of human cognition such that the processes of reflecting objective reality are based on the integrated representation of the studied object from the point of view of achieving the research goals, based on knowledge, experience, and foresight. 
Again, why would this be peculiar to systemics? Doesn't the causal modeller share this perspective as well?

On the one hand, soft and critical system thinking lost what made system science significantly different from 'classical' science, namely its metaphysics. On the other, a detailed systemic methodology to analyse data sets in social science is still lacking and when one is offered, it eventually boils down to the known methods of 'classical' science. The result is a position that claims a difference without offering any convincing argument for it.

Berlinski (1978) even came to attack system science saying that it is a "sham". The reason is this: the gap between its aspirations and its achievements is too large. Berlinski's line of argument is as follows. General system theory aims to provide those principles that are applicable to systems in general. This corresponds to Zgurovsky and Pankratova's metamethodology. However, if systems are merely described by ordinary differential equations, then those principles will simply correspond to the differential equations used in a given field, for instance the equation $F=m \frac{d v}{d t}$ in mechanics, or the law of the logistic curve in the theory of biological growth (see Berlinski 1978). But these are empirical laws certainly not valid for systems in general. Therefore system theory fails to meet the level of generality it aims to. It is worth noting that this general aspect constitutes a major goal of system science still nowadays (on this points see Bailey 2001, 2004, 2005). Consequently, Berlinski's criticism is still cogent, some thirty years later.

Nevertheless, I want to argue that there is still room for systemics, notably as a post hoc comparative tool. For a given phenomenon, causal analysis will identify the environment (i.e., the causal context, out of background knowledge), the elements (i.e., the most relevant variables to include into the causal model), and the structure (i.e., how those variables interrelate-the mechanism). This basically corresponds to the hypothetico-deductive methodology sketched in section 3 . The identification and (dis)confirmation of the structure is done within a causal context. Background knowledge is essential in a first stage where the context is identified and variables are chosen, as well as in a later stage where the structure is tested. It is also worth remembering that I pointed out the flexibility of the H-D methodology: 'negative' results may lead to change the model, to redefine conceptual variables, and may also lead to discard background knowledge itself. Notice that the order in which environment, elements, and structure are identified in the H-D methodology differs from the systemics' order. There, the elements are identified first, then the environment and finally the structure.

For a given phenomenon, systemic analysis will provide a more detailed picture, by suggesting more possible ways in which the elements of the system may interrelate. This results from the methodology proper to systemics. In fact, in choosing first the elements, the system analyst is not, in principle, limited by the context and by the available data. Having virtually more elements to 'play with' and a concept of system where every element interacts with everything else, the system analyst can now envisage a complex structure where as many relations as possible take place among the elements previously identified. 
Systemics will play the role of a post hoc comparative tool in the following sense. The question to be answered will be: is the causal story an adequate simplification of the systemic story? The answer to this question will depend, in part, on the purpose of the causal analysis, that is explanation, prediction, or policy. Also, as a post hoc comparative tool systemics will force causal modellers to give stronger theoretical, methodological, and empirical justifications to their modelling strategy and results. Loriaux and Franck suggested that a causal analysis find its place within system analysis. What I am suggesting, instead, is that once a causal analysis is completed, systemics play the role of an additional 'sounding board'.

Consider again the case study on health system and mortality in Spain discussed earlier. The objective of this study, recall, was to provide an explanation of a decline in regional mortality rates in the Eighties after socio-economic policies in the Seventies. What role would systemics have as a post hoc comparative tool? Let us follow systemic methodology.

First: identification of the elements. Lopez-Rios et al. (1992) identified six variables (including 'mortality' which explicitly plays the role of the effect). Loriaux (1994), instead, identified nineteen groups of concepts. We'd then expect the causal modeller to provide a sound justification for her choice. For instance, data allowed to measure 'economic development' but not 'inequalities between classes' (inégalités entre classes, in Loriaux's graph). Or, 'economic development' conceptually corresponds to the two joint boxes up-left in Loriaux's graph (structure de production et développement économique /état de la technique, structure industrielle,...) and is measurable with the available data, etc.

Second: identification of the structure. Loriaux complained, for instance, that according to well established economic theories 'economic development' generates 'social development' but examples where the relation is reversed exist. Here, the causal modeller ought to justify the direction of this bit of her mechanism. For instance, data allowed time ordering of the variables. Or, the causal modeller could invoke background knowledge explaining why in this case the relation goes from 'economic development' to 'social development' and not the other way round. A reason might be that policies in the Seventies in Spain mainly intervened on economic aspects.

Third: identification of the environment. Whilst system analysts get at the environment through the identification of the elements and of the structure, causal modellers take it as their starting point. It seems to me that concerning this last point causal modelling will be the sounding board of system analysis, rather than the reverse. The causal modeller has to clearly specify at the beginning of her analysis the population of reference and the socio-demo-political context. Will the system analyst identify the same population of reference using a priori elements and structures? I have already highlighted a tension in the systemic methodology in this 
respect: how could Loriaux have possibly selected the elements he did without using any background knowledge? The system analyst might rebut that what Loriaux drew is a general systemic story, not specific to Spain in the mid-Eighties. That's fine, but then the system analyst has to justify why her story is also applicable to the Spanish situation under analysis. It is in fact far from being selfevident that such a general scheme such as fig. 4 will automatically be valid in a specific case. As is well know to any social scientist, concepts and relations are highly context-relative. For instance, maternal education does not play the same role for child survival in developing and in developed countries.

Agreed, the use of systemics as a post hoc comparative tool just sketched is, at present, just a possibility to integrate two different approaches but never done in practice. This might be somewhat idealistic, as it would require involving a system scientist in every causal analysis. Idealistic, perhaps, but, to echo Wunsch (2007), as desirable as having a philosopher in every research group in science.

Of course, using system analysis as a post hoc comparative tool leads to abandon the strong metaphysical tenets of systemics. In particular, this leads to a charitable interpretation of systemics, where 'system' is given a weak connotation: causal mechanisms are within larger systems, but the postulate that every thing interacts with everything else is relaxed. Also, the notion of 'interaction' is given a weak and diversified interpretation: first, the agent interacts with the system in the sense that she interprets it but does not, ipso facto, modify it, and second, elements in the system can have mere correlational relations or causal interactions.

Soft and especially critical system thinkers would possibly not object to this move. Yet, this might be perceived as a defeat by hard system theorists seriously preaching a new course in science and in philosophy. But perhaps this just contributes to putting systemics in (the right) perspective. Herbert Simon, who defended and promoted systemics and cybernetics as early as 1969, seemed to have a reasonable and moderate view about the pretended change of paradigm. I'll leave Simon (1982 [1969], p. 193) the last word:

\footnotetext{
A number of proposals have been advanced in recent years for the development of 'general system theory' that, abstracting from properties peculiar to physical, biological, or social systems, would be applicable to all of them. We might feel that, while the goal is laudable, systems of such diverse kinds could hardly be expected to have any nontrivial properties in common. Metaphor and analogy can be helpful, or they can be misleading. All depends on whether the similarities the metaphor captures are significant or superficial.
}

\section{Acknowledgements}

I wish to thank Phyllis McKay, Jon Williamson, Guillaume Wunsch, and the participants of the seminars at the Centre for Reasoning (University of Kent, February 2008) and of the IUC Conference (Dubrovnik, April 2008) for stimulating and helpful comments on an earlier draft of this paper. Financial support from the FSR-FNRS (Belgium) is also gratefully acknowledged. 


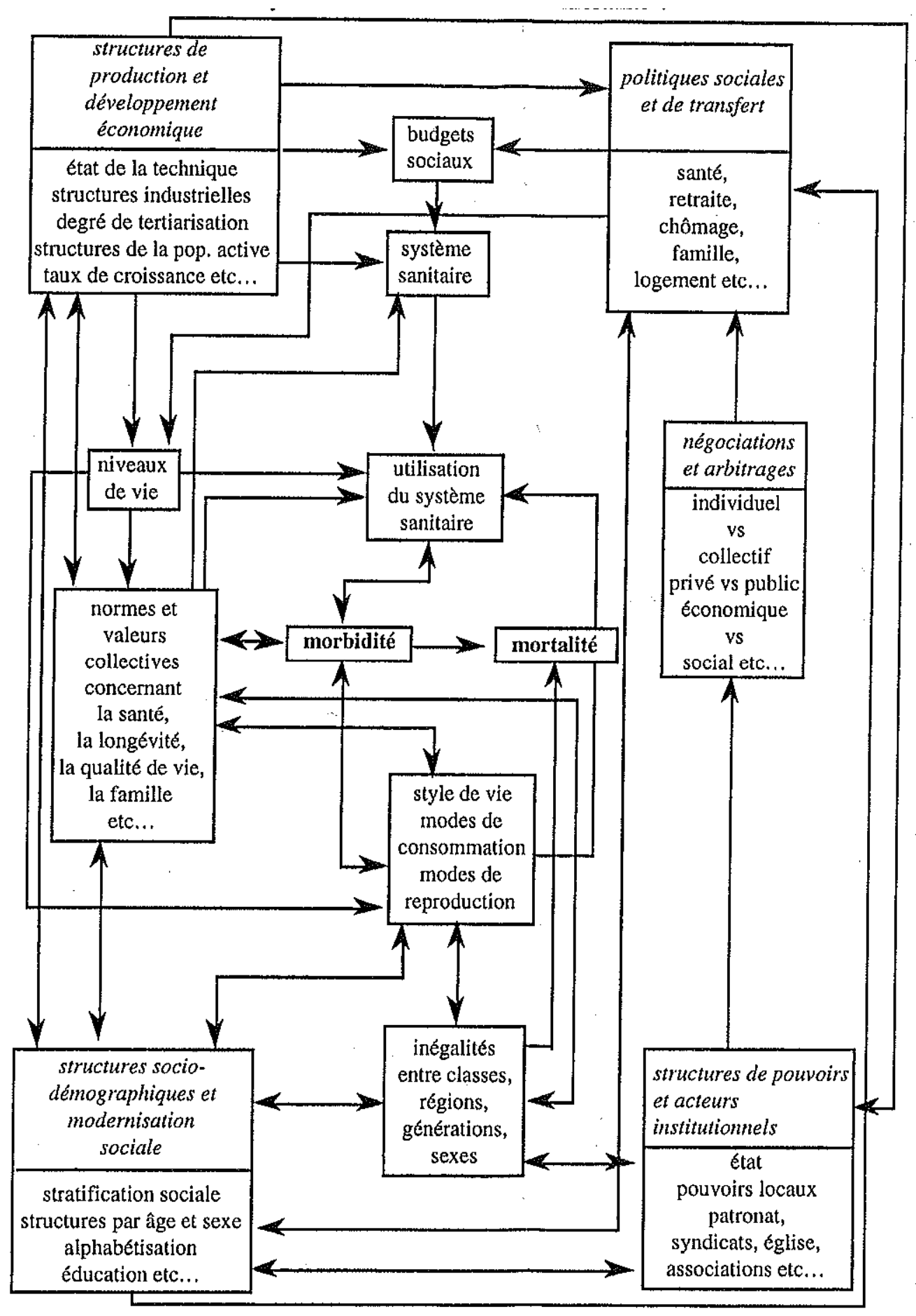

FIG. 4 Loriaux's systemic graph 


\section{References}

Aoki, M. (1984). System sciences and the modelling and analysis of dynamic economic phenomena. In A. Ruberti (Ed), System sciences and modelling (113138), Paris: Unesco.

Bailey, K. D. (2001). Towards unifying science: applying concepts across disciplinary boundaries. System Research and Behavioral Science, 18, 41-62.

Bailey, K. D. (2004). Incoming presidential address. Fifty years of system science. General bulletin systems bulletin, volume 33.

http://isss.org/bulletins/2004 GeneralSystemsBulletin v33 no member list.pdf Accessed 25 June 2008.

Bailey, K. D. (2005). Fifty years of system science: further reflections. System Research and Behavioral Science, 22, 355-361.

Berlinski, D. (1978). Adverse notes on system theory. In G. Klir (Ed), Applied general system research (949-960), New York: Plenum Press.

Bunge, M. (1977). The furniture of the world. Treatise on basic philosophy, Ontology 1. Dordrecht: Reidel Publishing Company.

Bunge, M. (1979), A world of systems. Treatise on basic philosophy, Ontology 2. Dordrecht: Reidel Publishing Company.

Bunge, M. (2000). Systemism: the alternative to individualism and holism. Journal of Socio-Economics, 29, 147-157.

Cartwright, N. (2007). Hunting causes and using them: approaches in philosophy and economics. Cambridge: Cambridge University Press.

Checkland P. (1995). Systems thinking, systems practice. Chichester: Wiley.

Deutsch, R. (1983). System analysis techniques. New York: Wiley.

Flood R. L., Jackson M. C. (Ed) (1991). Critical systems thinking. Chichester: Wiley.

Franck, R. (Ed) (1994). Faut-il chercher aux causes une raison? Paris: Vrin.

Franck, R. (Ed) (2002). The explanatory power of models. Dordrecht: Kluwer.

Franck, R. (2007). "Peut-on accroître le pouvoir explicatif des modèles?". In A. Leroux, P. Livet (Ed), Leçons de philosophie économique, Economica, Paris.

Freedman, D. A. (2005). Statistical models. Theory and practice. Cambridge: Cambridge University Press.

Little, D. (1998). Microfoundations, method, and causation. Essays in the philosophy of the social sciences. London: Transaction.

Lopez-Rios, O., Mompart, A., Wunsch, G. (1992). Système de soins et mortalité régionale: une analyse causale. European Journal of Population, 8(4), 363-379.

Loriaux, M. (1994). Des causes aux systèmes: la causalité en question. In R. Franck (Ed), Faut-il chercher aux causes une raison? (41-86). Paris: Vrin

Mingers, J. (2006). Realising system thinking: knowledge and action in management science. New York: Springer.

Mouchart, M., Russo, F., Wunsch, G. (2008). Structural modelling, exogeneity and causality. In H. Engelhardt, H-P Kohler, A. Prskwetz (Ed.), Causal analysis in population studies: concepts, methods, applications (chapter 4). Dordrecht: Springer.

Pearl, J. (2000). Causality. Models, reasoning, and inference. Cambridge: Cambridge University Press.

Popper, K. (1959). The logic of scientific discovery. London: Hutchinson. 
Ruberti, A. (Ed) (1984). System sciences and modelling. Reidel Publishing Company.

Russo, F. (2008). Causality and causal modelling in the social sciences. Measuring variations. Springer. In press.

Salmon, W. C. (1984). Scientific explanation and the causal structure of the world. Princeton: Princeton University Press.

Salmon, W.C. (1990). Causal propensities: statistical causality vs. aleatory causality. Topoi, 9, pp. 95-100.

Simon, H. A. (1982) [1969]. The sciences of the artificial. Cambridge, Mass.: MIT Press.

Spirtes, P., Glymour, C., Scheines, R. (1993). Causation, Prediction, and Search. New York, N.Y.: Springer-Verlag. 2nd Edition, MIT Press (2001).

von Bertalanffy, L. (1968). General system theory: foundations, development, applications. New York: Braziller.

Wunsch, G. (1988). Causal theory and causal modelling. Leuven: Leuven University Press.

Wunsch G. (2007). Interview with Michel Mouchart and Guillaume Wunsch. The Reasoner, vol 1 (8), p.3. http://www.thereasoner.org/, accessed 7 July 2008.

Zgurovsky, M. Z., Pankratova, N. D. (2007). System analysis: theory and applications. Berlin-Heidelberg: Springer-Verlag. 